\title{
New therapeutic avenue of epigenetic modulations in cancer
}

\author{
Desi Pan, Xianping Lu \\ Shenzhen Chipscreen Biosciences Co., Ltd., Shenzhen 518057, China \\ Correspondence to: Dr. Xianping Lu. Shenzhen Chipscreen Biosciences Co., Ltd., Shenzhen 518057, China. Email: xplu@chipscreen.com. \\ Provenance and Peer Review: This article was commissioned by the Editorial Office, Translational Breast Cancer Research. The article was sent for \\ external peer review.
}

Received: 24 February 2020; Accepted: 24 March 2020; Published: 10 April 2020.

doi: $10.21037 /$ tbcr.2020.03.03

View this article at: http://dx.doi.org/10.21037/tbcr.2020.03.03

Epigenetics was firstly introduced by Conrad Waddington as a term to explain why the phenotypic variations due to other factors rather than genetic and currently refers to the study of mitotically and/or meiotically heritable changes in gene expression that occur without changes in the DNA sequence. Up to date, epigenetic regulation expresses in multiple forms that include DNA methylation, covalent histone modifications (methylation, acetylation, phosphorylation, etc.), chromatin modelling, and microRNA expression. The major corresponsive proteins or enzymes that modify the substrates include DNA methyltransferases (DNMT1, DNMT3A, DNMT3B) and DNA demethylase (ten-eleven translocation family proteins, TETs) for DNA methylation; histone methyltransferases (DOT1L, EZH2, MLL, etc.) and histone demethylases (LSD1-2, KDM2-8, etc.) for histone methylation; protein lysine acetyltransferases (Gcn5/PCAF, p300/CBP, and MYST) and histone deacetylases (HDAC1-11, SIRT1-7) for acetylation of substrates such as histones, transcription factors and other proteins; chromatin remodeling ATPase families (SWI/SNF, ISWI, NuRD/Mi-2/CHD, and INO80); and plant homeodomain zinc fingers (PHD) and bromodomain and extra-terminal (BET) proteins that interact with gene transcription machinery via binding to modified chromatin, etc. (1).

Modifications to DNA and histones play a critical role in the regulation of all DNA-based processes, such as transcription, DNA repair, and replication. Generally, they are executed in a highly regulated manner during development and differentiation processes in body. However, the dysregulation and altered epigenetic status are common in many diseases, from metabolic diseases to various cancers (2). Many efforts in drug discovery for cancer therapy by targeting variety of epigenetic modifiers have been taken in recent years because the contributions of epigenetic alterations in cancer growth, development, recurrence and metastasis, especially against tumor cell heterogeneity, cancer stem cell, plasticity of tumor cells and repressed tumor microenvironment, are getting evident and clear (3). Those characteristics of epigenetic alterations in cancer may open a new avenue in seeking novel treatments against eventually recurrent/relapse, metastasis and drug resistance in cancer patients whom otherwise would fail in obtaining extended benefits of overall survival.

\section{Epigenetic drugs in hematologic malignancies}

Epigenetic therapy in hematological malignancies is the most advanced field with a confirmed potential. Hematopoiesis includes multi-step and multi-lineage development process from hematopoietic stem cell progenitors (HSCs) to terminal differentiated cell types including platelet, erythrocyte, granulocyte, monocyte, and lymphocyte, etc. Epigenetic status and transcription program are dramatically changed in transient or permanent but in well-controlled manner during these processes. Malignancies would arise in any development process if the homeostasis goes wrong (4).

The cytidine analogs, 5-azacytidine and decitabine first appeared as chemotherapeutic agents against acute leukemia, which early studies showed anti-leukemic efficacy at relatively high doses however with pronounced toxicity. It was later proved to execute clinical efficacy at lower dose as an epigenetic drug by reducing DNA methylation rather than direct cytotoxic agent. Both have been approved for treatment in patients with myelodysplastic syndrome (MDS) and elderly patients with acute myeloid leukemia (AML) (5).

Similar to DNA hypomethylating agents, another major 
epigenetic drug class histone deacetylase (HDAC) inhibitor was firstly recognized with its differentiation promoting effect in AML cells. HDACs could regulate acetylation of both histones and transcription factors via interaction with many other transcriptional complexes leading to repression of transcription, for instance, genetic lesions in AML such as fusion proteins AML1-ETO, PML-RAR $\alpha$ and RAR $\alpha-$ PLZF could repress genes transcription responsible for hematopoietic differentiation via recruitment of HDAC1 and 3 that results in leukemogenesis (6). So far there are already five HDAC inhibitors approved, vorinostat for cutaneous $\mathrm{T}$ cell lymphoma, romidepsin, belinostat and tucidinostat (chidamide) for peripheral $\mathrm{T}$ cell lymphoma, and panobinostat in combination with bortezomib and dexamethasone for multiple myeloma (7).

Although many epigenetic related genetic lesions have been found common in hematologic malignancies, it was noticeable that current DNMT or HDAC inhibitors were approved to generally use in patients without a definitive correlation with regarding to the epigenetic mutations. It is mostly due to limited understanding of the intrinsic pathogenic roles of epigenetic alterations among tumor types up to date. One exception is isocitrate dehydrogenases (IDH). Mutant IDH1 and IDH2 which found in about 20\% of AML patients, could produce more oncometabolite 2-hydroxyglutarate which interferes with DNA methylation and block cell differentiation. There are already two IDH inhibitors approved, IDH1 inhibitor ivosidenib for the treatment of relapsed or refractory AML with a susceptible IDH1 mutation, and IDH2 inhibitor enasidenib for the treatment of relapsed or refractory AML with an IDH2 mutation (8).

Except broad impact on cellular function and phenotype by epigenetic modifications, there are also particular interactions of oncogenic factors with HDAC signaling dependent on cellular context (9). For instance, antiapoptotic protein $\mathrm{Bcl}-2$ and oncogenic c-Myc were repressed by the selective HDAC inhibitor tucidinostat in preclinical model of diffuse large B-cell lymphoma (DLBCL). Both proteins are over expressed in tumors of a portion of DLBCL patients with poorer prognosis with the existing treatments. In a phase 2 clinical study in elderly patients with DLBCL that included significant portion of double expressed patients (Bcl-2 and c-Myc), tucidinostat in combination with standard regime of 6 cycles of R-CHOP-21 (addition of rituximab to cyclophosphamide, doxorubicin, vincristine and prednisolone given every 21 days) showed promising activity, obtained complete response (CR) of $85.4 \%$ among 41 evaluable patients and one year progress free survival (PFS) of $92.1 \%$ after a median follow-up of 18 months. Interestingly similar efficacy was found in patients defined as Bcl-2 and c-Myc double expressed (10). Based on the data and concurrent clinical observations from other IIT studies, the pivotal phase 3 study of tucidinostat in combination with R-CHOP in patients with newly diagnosed double-expressed DLBCL has been initiated in china (NCT04231448). However, in one phase 2 trial using a pan HDAC inhibitor panobinostat in combination with rituximab for treatment in heavily pretreated patients with DLBCL, only moderate responses were reported (11). Although selective HDAC inhibitor tucidinostat has been shown with activity in DLBCL in previous monotherapy studies, further optimized combinatory regimens and patient stratifications need to be defined based on different biological characteristics of patients.

\section{Epigenetic drugs in solid tumors}

Unlike hematologic malignancies, there are significantly more complicated heterogeneity and microenvironment within solid tumors (12). In previous clinical studies with epigenetic drug monotherapy in patients with solid tumors, there are only moderate or no objective response reported. One critical point to consider is that epigenetic drugs not designed to kill tumor cells like a cytotoxic agent instead to normalize tumor cell phenotype and rebuild tumor microenvironment, which may essentially enhance tumor response to other therapies. For instance, HDAC inhibitors vorinostat, entinostat and tucidinostat have shown synergic effect with endocrine therapy via multiple mechanisms in preclinical studies on breast cancer, including restore of expression of silenced estrogen receptor (ER)- $\alpha$, downregulation of estrogen-independent growth factor signaling pathway (epidermal growth factor receptor/MEK/ERK), inhibition of phosphorylation and activation of ER $\alpha$, etc., rendering sensitivity to hormonal therapy. HDAC inhibitors such as tucidinostat or entinostat in combination with ERa antagonist fulvestrant or aromatase inhibitor letrozole, showed synergic tumor suppression in $\mathrm{ER} \alpha$ positive breast cancer xenograft models $(13,14)$.

In a phase 2 study, entinostat was compared with placebo in existence of aromatase inhibitor exemestane in patients with ER positive breast cancer progressed from prior endocrine therapy. Entinostat showed trend in improving the primary endpoint PFS (4.3 versus 2.3 months with placebo) although not statistically significant $(\mathrm{P}=0.055)$. Meanwhile entinostat obtained significant improvement 
in secondary end point overall survival (OS), which was 28.1 months with entinostat versus 19.8 months with placebo ( $\mathrm{P}=0.036)$ (15). Recently, Syndax Pharmaceuticals announced the successful interim futility analysis of overall survival data, one primary endpoint of its phase 3 clinical trial E2112, which evaluating the combination of entinostat and exemestane compared with placebo plus exemestane in patients with advanced hormone receptor-positive and Her2 negative breast cancer, although no significance in another primary endpoint PFS achieved according to a previous report, and the trial is continued to its OS data matured (Sourced from: https://www.prnewswire.com/newsreleases/syndax-pharmaceuticals-reports-third-quarter2019-financial-results-and-provides-clinical-and-businessupdate-300954227.html. Accessible on Feb 15, 2020).

In an exploratory single arm phase 2 trial, tucidinostat in combination with exemestane obtains objective response rate of $25 \%$ in 16 evaluable patients with advanced hormone receptor-positive and Her-2 negative breast cancer recurrent or progressed from at least one prior endocrine therapy. The median PFS was 7.6 months (16). In the phase 3 ACE study, tucidinostat plus exemestane is well tolerated and significantly improve PFS in patient with advanced hormone receptor-positive and Her-2 negative breast cancer progressed after previous endocrine therapy by comparing with placebo plus exemestane $(7.4$ versus 3.8 months in the placebo group, $\mathrm{P}=0.033$ ). The addition of tucidinostat to exemestane also obtained a higher objective response rate than that with placebo $(16 \%$ versus $7 \%, \mathrm{P}=0.024)$. Overall survival results were not mature at the data cutoff date and study is continued until survival results are mature for analysis (17).

The ACE study has provided important insight into the potential of targeting epigenetic signaling to overcome antiestrogen resistance. Tucidinostat has already approved by the National Medical and Pharmaceutical Administration (NMPA) in China for treatment in patients with hormone receptor-positive metastatic breast cancer. HDAC inhibitor such as tucidinostat could thus emerge as a new therapeutic tool in the rapidly evolving landscape of combined targeted therapies against drug resistance, tumor relapse and reactivation of microenvironment (18).

On the other hand, the main challenge in application of epigenetic drugs in solid tumors is still lack of knowledge about the intrinsic roles of epigenetic aberrations in individual tumor type. Recently, tazemetostat which target a histone methyltransferase EZH2 (enhancer of zeste homolog 2) has succeeded in epithelioid sarcoma (ES), a rare form of soft tissue sarcoma (STS). Loss of integrase interactor 1 (INI1) which is a subunit of the chromatin remodeling family member SWI/SWF complex and a negative regulator of $\mathrm{EZH} 2$, occurs in over $90 \%$ of ES. In a phase 2, open-label, multi-center study, tazemetostat was tested in patients with ES and confirmed with tumor bearing INI1 loss. The drug produced objective response rate of $15 \%$, which met the primary end point of this trial, and $67 \%$ of the responses lasted at least 6 months. The FDA has approved tazemetostat for treating ES as the first targeted treatment for ES as a single agent (19). It is the first epigenetic drugs approved as monotherapy in solid tumor with specific genetic lesion.

\section{Lessons from the past and future directions}

Tucidinostat and tazemetostat respectively represent two promising approaches to develop epigenetic drugs in solid tumors, either to promote efficacy of existing therapies via synergic effects, or to target particular epigenetic lesions leading to tumor initiation or progression.

As known, epigenetic modifications are highly involved in DNA damage repair and cell cycle control (20). With overall satisfactory tolerance of epigenetic drugs, it is reasonable to combine epigenetic drugs with chemotherapies, radiotherapy, and other targeting therapies under the specific clinical circumstances like drug resistance, treatment relapse and metastasis. In a realworld study of tucidinostat in total of 1,064 patients with relapsed or refractory PTCL, tucidinostat in combination with investigator chosen conventional chemotherapy regimens (CHOP, GDP, or EPOCH) indeed increase clinical response $(65.4 \%$ versus $47 \%$ with tucidinostat monotherapy) and overall survival (15 versus 11.2 months with tucidinostat monotherapy) [Personal communication. Ma J, Zhu J, Shi Y, et al. Presented on the $11^{\text {th }} \mathrm{T}$ Cell Lymphoma Forum (TCLF), Jan 30-Feb 1, 2019, La Jolla, CA, USA]. HDAC inhibitor such as vorinostat has been tested in head and neck squamous cell carcinoma (NCT01064921) and glioblastoma (NCT00731731) in combination with chemoradiation therapy and obtained positive implications in clinical response or overall survival, although further investigations are strongly warranted.

Both DNMT inhibitors and HDAC inhibitors, most evidently the selective HDACi such as tucidinostat and entinostat, can increase the immunogenicity of tumor cells by promoting the expression of cancer testes antigens and MHC I antigen presenting machinery. HDAC inhibitor 


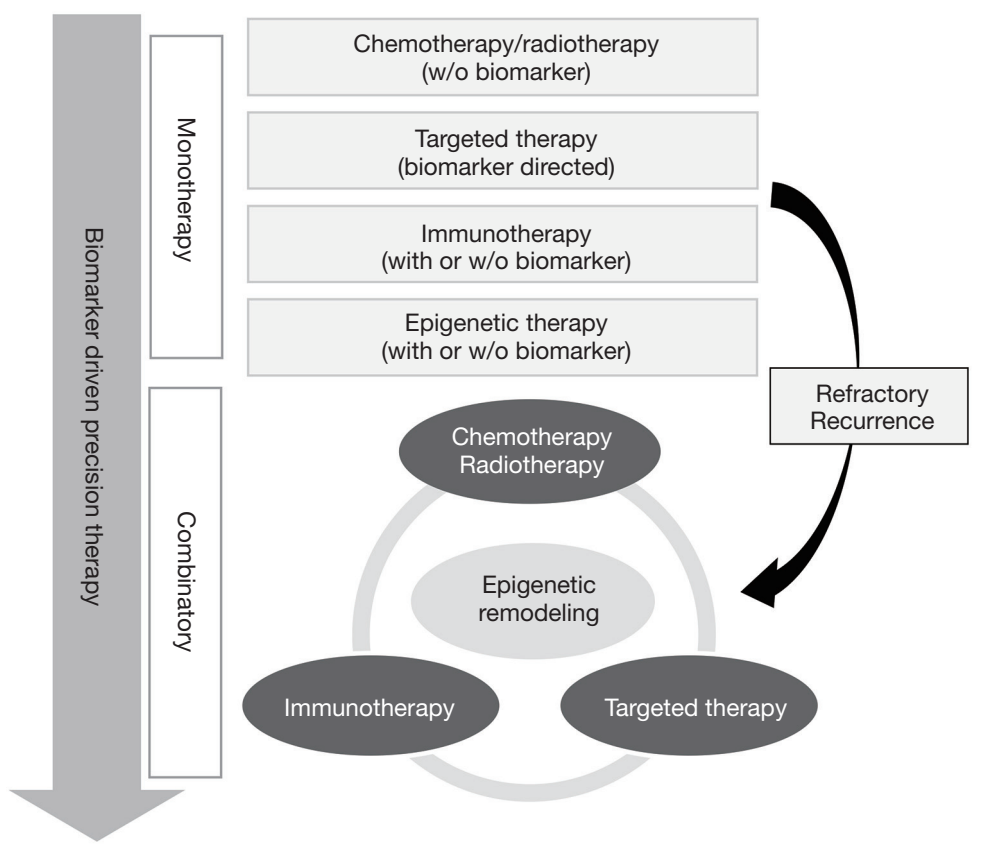

Figure 1 Epigenetic strategy in the roadmap of cancer therapy.

tucidinostat was also demonstrated to enhance natural killer (NK) and cytotoxic T lymphocyte (CTL) mediated antitumor immunity, and an intact host immune system was showed to be essential for inducing sustained anticancer responses against solid and haematological tumours by HDAC inhibitors (21). Indeed, there are many active clinical trials combining epigenetic drugs and immune therapy opened in the clinicaltrials.gov database, including DNMT inhibitors (azacitidine, decitabine, guadecitabine, CC-486), HDAC inhibitors (vorinostat, panobinostat, entinostat, tucidinostat, mocetinostat, domatinostat), EZH2 inhibitors (tazemetostat, CPI-1205), and BET inhibitors (BMS-986158), etc. (22).

Several positive preliminary results have been posted from those combination approaches. For instance, a phase 2 open label study of decitabine plus PD-1 blocker camrelizumab produced significantly higher CR rate at $71 \%$ versus $32 \%$ with camrelizumab monotherapy in 86 patients with relapsed/refractory classical Hodgkin lymphoma (23); a phase 2 study of entinostat in combination with PD-1 blocker pembrolizumab obtained $10 \%$ of objective response rate in patients with NSCLC who had progressed on/after anti-PD-1/PD-L1 therapy (24); similarly, a phase $1 \mathrm{~b} / 2$ study of tucidinostat plus nivolumab reported a $38 \%$ of objective response rate in NSCLC patients progressed from prior therapy with or without PD-(L)1 blocker (25). More clinical studies are ongoing with combination of epigenetic drugs and immunotherapy.

For breast cancer, a previous phase 2 study of DNMT inhibitor 5-azacitidine in combination with HDAC inhibitor entinostat did not met the primary endpoint although well tolerated in patients with advanced hormoneresistant or triple-negative breast cancer (TNBC) (26). There are also two ongoing clinical trials for tucidinostat, one is a prospective real world study of tucidinostat in combination with fulvestrant, anti-Her-2, or PD-1 blocker for patients with ER positive, Her-2 positive or Triplenegative advanced breast cancer respectively (registration number: ChiCTR2000029509), another is a phase 2 clinical trial of tucidinostat in combination with cisplatin for patients with relapsed or metastatic triple-negative breast cancer (NCT04192903). The benefits of addition of epigenetic drugs into variety of combinatory treatment regimens for diverse group of patients with advanced breast cancer are still needed to be clarified.

Given the great advancements in our understanding of epigenetic pathways and clinical development of variety of epigenetic drugs, the avenue for clinical applications of this new therapeutic class is widely open, either as monotherapy or in combination with other regimes (Figure 1). 


\section{Acknowledgments}

Funding: None.

\section{Footnote}

Conflicts of Interest: Both authors have completed the ICMJE uniform disclosure form (available at http://dx.doi. org/10.21037/tbcr.2020.03.03). The authors have no conflicts of interest to declare.

Ethical Statement: The authors are accountable for all aspects of the work in ensuring that questions related to the accuracy or integrity of any part of the work are appropriately investigated and resolved.

Open Access Statement: This is an Open Access article distributed in accordance with the Creative Commons Attribution-NonCommercial-NoDerivs 4.0 International License (CC BY-NC-ND 4.0), which permits the noncommercial replication and distribution of the article with the strict proviso that no changes or edits are made and the original work is properly cited (including links to both the formal publication through the relevant DOI and the license). See: https://creativecommons.org/licenses/by-nc$\mathrm{nd} / 4.0 /$.

\section{References}

1. Allis CD, Jenuwein T. The molecular hallmarks of epigenetic control. Nat Rev Genet 2016;17:487-500.

2. Flavahan WA, Gaskell E, Bernstein BE. Epigenetic plasticity and the hallmarks of cancer. Science 2017. doi: 10.1126/science.aal2380.

3. Easwaran H, Tsai HC, Baylin SB. Cancer epigenetics: tumor heterogeneity, plasticity of stem-like states, and drug resistance. Mol Cell 2014;54:716-27.

4. Butler JS, Dent SY. The role of chromatin modifiers in normal and malignant hematopoiesis. Blood 2013;121:3076-84.

5. Yun S, Vincelette ND, Abraham I, et al. Targeting epigenetic pathways in acute myeloid leukemia and myelodysplastic syndrome: a systematic review of hypomethylating agents trials. Clin Epigenetics 2016;8:68.

6. Wang P, Wang Z, Liu J. Role of HDACs in normal and malignant hematopoiesis. Mol Cancer 2020;19:5.

7. Moskowitz AJ, Horwitz SM. Targeting histone deacetylases in T-cell lymphoma. Leuk Lymphoma 2017;58:1306-19.
8. Contieri B, Duarte BKL, Lazarini M. Updates on DNA methylation modifiers in acute myeloid leukemia. Ann Hematol 2020. doi: 10.1007/s00277-020-03938-2.

9. Li Y, Seto E. HDACs and HDAC Inhibitors in Cancer Development and Therapy. Cold Spring Harb Perspect Med 2016. doi: 10.1101/cshperspect.a026831.

10. Zhang M, Fang Y, Xu P, et al. Chidamide Plus R-CHOP21 in Elderly Patients with Newly Diagnosed Diffuse Large B-Cell Lymphoma: Results of a Phase II Study. Blood 2018;132:2968.

11. Barnes JA, Redd R, Fisher DC, et al. Panobinostat in combination with rituximab in heavily pretreated diffuse large B-cell lymphoma: Results of a phase II study. Hematol Oncol 2018;36:633-7.

12. McGranahan N, Swanton C. Clonal Heterogeneity and Tumor Evolution: Past, Present, and the Future. Cell 2017;168:613-28.

13. Sabnis GJ, Goloubeva OG, Kazi AA, et al. HDAC inhibitor entinostat restores responsiveness of letrozoleresistant $\mathrm{MCF}-7 \mathrm{Ca}$ xenografts to aromatase inhibitors through modulation of Her-2. Mol Cancer Ther 2013;12:2804-16.

14. Zhou Y, Wang YN, Zhang K, et al. Chidamide reverses epidermal growth factor induced endocrine resistance in estrogen receptor-positive breast cancer. J Shenzhen Univ Sci Eng 2018;35:339-44.

15. Yardley DA, Ismail-Khan RR, Melichar B, et al. Randomized phase II, double-blind, placebo-controlled study of exemestane with or without entinostat in postmenopausal women with locally recurrent or metastatic estrogen receptor-positive breast cancer progressing on treatment with a nonsteroidal aromatase inhibitor. J Clin Oncol 2013;31:2128-35.

16. Zhang Q, Wang T, Geng C, et al. Exploratory clinical study of chidamide, an oral subtype-selective histone deacetylase inhibitor, in combination with exemestane in hormone receptor-positive advanced breast cancer. Chin J Cancer Res 2018;30:605-12.

17. Jiang Z, Li W, Hu X, et al. Tucidinostat plus exemestane for postmenopausal patients with advanced, hormone receptor-positive breast cancer (ACE): a randomised, double-blind, placebo-controlled, phase 3 trial. Lancet Oncol 2019;20:806-15.

18. Wander SA, Spring LM, Bardia A. Genetics to epigenetics: targeting histone deacetylases in hormone receptorpositive metastatic breast cancer. Lancet Oncol 2019; 20:746-8.

19. Gounder M, Schöffski P, Villalobos V, et al. 1615PD: 
A phase II, multicenter study of the EZH2 inhibitor tazemetostat in adults: Epithelioid sarcoma cohort (NCT02601950). Ann of Oncol 2018. doi: 10.1093/ annonc/mdy299.014.

20. Potenski CJ, Klein HL. Molecular biology: The expanding arena of DNA repair. Nature 2011;471:48-9.

21. Maio M, Covre A, Fratta E, et al. Molecular Pathways: At the Crossroads of Cancer Epigenetics and Immunotherapy. Clin Cancer Res 2015;21:4040-7.

22. Olino K, Park T, Ahuja N. Exposing Hidden Targets: Combining epigenetic and immunotherapy to overcome cancer resistance. Semin Cancer Biol 2020. doi: 10.1016/ j.semcancer.2020.01.001.

23. Nie J, Wang C, Liu Y, et al. Addition of Low-Dose Decitabine to Anti-PD-1 Antibody Camrelizumab in Relapsed/Refractory Classical Hodgkin Lymphoma. J Clin

doi: 10.21037/tbcr.2020.03.03

Cite this article as: Pan D, Lu X. New therapeutic avenue of epigenetic modulations in cancer. Transl Breast Cancer Res 2020;1:2.
Oncol 2019;37:1479-89.

24. Sullivan RJ, Moschos SJ, Johnson ML, et al. Abstract CT072: Efficacy and safety of entinostat (ENT) and pembrolizumab (PEMBRO) in patients with melanoma previously treated with anti-PD1 therapy. Cancer Res 2019;79:CT072.

25. Bissonnette R, Khushalani N, Eroglu Z, et al. P346: A phase $1 \mathrm{~b} / 2$ study of the safety and efficacy of HBI-8000nivolumab combination in melanoma (MEL), renal cell carcinoma (RCC) and non-small cell lung cancer (NSCLC). J Immunother Cancer 2018;6:P346.

26. Connolly RM, Li H, Jankowitz RC, et al. Combination Epigenetic Therapy in Advanced Breast Cancer with 5-Azacitidine and Entinostat: A Phase II National Cancer Institute/Stand Up to Cancer Study. Clin Cancer Res 2017;23:2691-701. 
1. Dr. Lu has mentioned many epigenetic modulations, DNMT, HDAC or EZH2, in bematologic malignancies. What are the prospects of these drugs in solid tumors? Moreover, what are the specific differences and similarities in the mechanisms or targets among these HDAC inbibitors like entinostat, tucidinostat?

Author's reply:

Our understanding about epigenetic machinery is still evolving even as today in biomedical research community. The machinery is basically composed of writers (DNA methylation, histone acetylation and many other chemical modifications), eraser (demethylation, deacetylation, etc.), readers (to read those chemical modifications in DNA or proteins), movers (to remodel chemical modifications in space) and shapers (construction of spatial configuration for modifications). However, the precise mechanism of actions for each of these machineries in relation to cancer development is far from understood. Luckily hematologic malignancies been first kind of clinic application by epigenetic modulators were the results of discovery of critical roles of the above-mentioned machinery in governing hematological stem cell differentiation and development, which the aberrations or mutations from the machinery became the driven force in hematologic malignancy.

It is less than 10 years that the roles of these machinery in solid tumors start to emerge, namely control of specific sets of cell fates in heterogeneity and evolution of tumor, plasticity of tumor in response to treatment, and immune surveillance via tumor's microenvironment beyond knowing the role of driver mutation to specific solid tumor as in the case of EZH2 inhibitor in ES.

These new discoveries are leading to new clinic strategy in the fight against tumor relapse/recurrent, treatment resistance, and relief of immune suppression. Therefore, it is expecting to see more and more clinic studies aiming to combine existing regimes with epigenetic modulators in attempt to significantly extend overall survival of cancer patients. In fact, large amount of clinical trials was conducted following such strategy and produced encouraged preliminary efficacy, for example, by combining with immune checkpoint inhibitors.

Both entinostat and tucidinostat are benzamide structure type that selectively inhibits class I HDAC 1,2 , and 3 subtypes with differential activity against these three individual subtypes. Further, tucidinostat also inhibits subtype 10 of class IIb HDAC. These two inhibitors however share more similarity than that with other HDAC inhibitor classes in biological activities especially in terms of activation of anti-tumor immune response. The major differences between those two inhibitors in human lie in their in vivo metabolism and pharmacokinetic profile where tucidinostat is able to archive optimal blood concentration that exercises epigenetic modulation over tumor cells as well as exhibits inhibitory activity against HDAC subtype 10 .

\section{It seems that there are no effective biomarkers to predict real efficacy of epigenetic modulations at present. The question is how to choose the right target population in the future exploration?}

\section{Author's reply:}

It is truly lack of reliable biomarkers to stratify patients with more likely benefit from epigenetic therapy currently. Both abnormal epigenetic modifications and genetic mutations in epigenetic enzymes have been tested as predictors of response to individual epigenetic drugs, however, the most challenge lies in the high failure rate of translation of those findings from relatively small sized exploratory studies to pivotal trials. Perhaps more approaches using omics-data in future prospective trials are needed to identify the right target population for this new drug class.

\section{We have known that HDAC inhibitors have been successful in ER positive breast cancer, which is also an important progress of epigenetic regulators in solid tumors. However, I wonder if there will be any potential benefits to other types of breast cancer from epigenetic regulators? If so, what type of breast cancer and what kind of epigenetic regulators will be?}

Author's reply:

Compared with other tumor types, breast cancer has less known driver genetic lesions, in the other words, there is no much targeted therapy for breast cancer except endocrine therapy, especially for triple negative breast cancer (TNBC) which relied on chemotherapy. It is better to apply epigenetic drugs as adjuvant to other regimen based on their regulatory roles in multiple growth pathways, for example, BET inhibitor may exerts synergistic effect with chemotherapy and targeted therapies via inhibition of $\mathrm{Myc}$ and Bcl-2 in both HR positive breast cancer or TNBC. As immunotherapy has generally low response rate in TNBC, epigenetic drugs such as DNMT or HDAC inhibitors 
could promote its efficacy by priming or reactivating the suppressive immune microenvironment. It has been studied that treatment of TNBC cells without BRCA mutations by HDAC inhibitor could generate a BRCAness in those cells, which leads to a synthetic lethality once combined with PARP inhibitor treatment. However, all those strategies need to be demonstrated with efficacy and tolerability in future clinical studies. 